\section{Regards sur l'économie allemande}

Bulletin économique du CIRAC

$66 \mid 2004$

Varia

\title{
Quelles infrastructures pour la Bohême au cœur de l'Europe?
}

Isabelle Bourgeois

\section{OpenEdition}

Journals

Édition électronique

URL : http://journals.openedition.org/rea/3862

DOI : 10.4000/rea.3862

ISBN : 978-2-8218-0828-7

ISSN : 1965-0787

Éditeur

CIRAC

Édition imprimée

Date de publication : 1 mai 2004

Pagination : 33-36

ISSN : 1156-8992

Référence électronique

Isabelle Bourgeois, "Quelles infrastructures pour la Bohême au cœur de l'Europe? », Regards sur l'économie allemande [En ligne], 66 | mai 2004, mis en ligne le 13 octobre 2009, consulté le 19 avril 2019. URL : http://journals.openedition.org/rea/3862 ; DOI : 10.4000/rea.3862 


\title{
Quelles infrastructures pour la Bohême au cœur de l'Europe?
}

\author{
Isabelle Bourgeois
}

Saxe, Bavière et Autriche partagent leurs frontières sur plus de $2000 \mathrm{~km}$ avec, du Nord au Sud: la Pologne, la République tchèque, la Slovaquie, la Hongrie et la Slovénie. Marges orientales de l'Europe occidentale durant de longues décennies - de la construction du Rideau de fer jusqu'à l'Unité allemande -, elles retrouvent leur place au centre de l'Europe en ce mois de mai 2004, avec l'arrivée de dix nouveaux Etats membres dans l'UE. Une région transfrontalière tout particulièrement pourrait désormais se transformer en un de ces pôles d'activités (clusters) indispensables à la compétitivité territoriale (voir REA 61/03) du nouvel espace européen : ce triangle que fait l'Ouest de la République tchèque avec la Saxe au Nord, la Bavière à l'Ouest et l'Autriche au Sud, et qui correspond pour l'essentiel à la Bohême, cœur historique de l'industrialisation européenne. Mais le potentiel de croissance de cette région est soumis à une condition : son désenclavement.

Pour les

entreprises

allemandes,

l'intégration

institutionnelle

des nouveaux

Etats membres

ne fait

qu'entériner

leur intégration

économique -

aujourd'hui

presque achevée, estime l'Institut IWH.

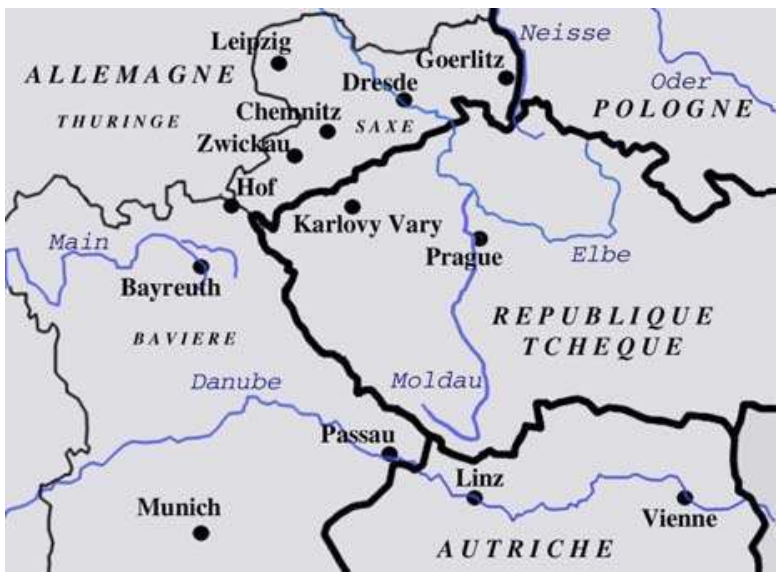

Les nouveaux entrants achètent à la RFA plus de $10 \%$ de ses exportations (autant que les USA), et ils vendent à l'UE entre $60 \%$ et $80 \%$ de leurs exportations. De tous, la République tchèque est le partenaire commercial de l'Allemagne avec lequel les échanges sont les plus diversifiés, le traitement passif cédant le pas aux produits et services à haute valeur ajoutée (voir REA 59/02). De tous, cet Etat est aussi celui qui attire le plus d'IDE allemands, avec près de 4,3 milliards $€$ en 2002 sur un total de 7 milliards $€$ investis dans les PECO (Bundesbank). Indépendamment de ses liens historiques (mais pas toujours exempts de crispation) avec la Saxe, la Bavière ou l'Autriche, comme le fort degré d'ouverture de son économie en comparaison des autres PECO ou la haute qualification de sa main-d'œuvre, sa position géographique centrale est un avantage compétitif pour la République tchèque. L'industrie automobile mondiale ne s'y est pas trompée, qui a égrené ses sites selon un axe nord-ouest/sud-est à partir de la Bavière et de la Saxe pour traverser la République tchèque (Skoda à Mladá Boleslav, par exemple), la Slovaquie (VW à Bratislava, à côté de Vienne) jusqu'à la Hongrie (Audi à Györ ou Suzuki à Estergom).
République tchèque : une économie bien placée 
L'urgence d'une politique d'infrastructures interrégionale

République tchèque : désengorger le trafic ouest-est ...

... et optimiser l'accès au port de Hambourg, au nord

Autriche : corridors vers l'est et extension du réseau intérieur

Le problème de l'aménagement du Danube
L'intégration plénière de cet Etat dans le marché intérieur rappelle l'importance des infrastructures de transport pour la dynamique de croissance des pôles d'activité déjà constitués ou en passe de s'installer dans leur sillage. Car avec la forte croissance des activités attendue dans ce pays et ses voisins, l'augmentation du trafic (fret, surtout) risque de conduire à un engorgement des grands axes existants et, parallèlement, de retarder dans leur développement économique les régions moins bien desservies. Or la réalité des politiques communautaire et nationales est loin de répondre aux besoins liés à l'élargissement. L'état des projets d'interconnexion dans cette région de Bohême aux multiples frontières en est symptomatique.

Si la République tchèque est l'Etat est-européen le plus proche de la moyenne ouest-européenne en termes d'équipements, la modernisation de ses infrastructures est loin d'être achevée, et leur extension comme leur raccordement aux réseaux voisins à l'Ouest reste largement à faire. Le gouvernement tchèque a programmé quelque 600 millions $€$ dans le budget 2004 pour l'entretien et la modernisation de son réseau routier et autoroutier. Dans le cadre de partenariats public/privé, la construction de deux grands axes autoroutiers est prévue : Prague-Nuremberg et Prague-Dresde, ce dernier tronçon (150 km) faisant partie du projet de corridor paneuropéen reliant Budapest, Sofia et Istanbul, en passant par Györ. La République tchèque est une importante plateforme pour les échanges ouest-est sur le continent européen.

Mais ces efforts ne suffiront pas à absorber l'augmentation prévisible du trafic de marchandises dont une large partie devrait quitter le rail pour la route, pour des raisons notamment de coûts, mais aussi de délais liés à l'évolution de la logistique (voir REA 49/00). Or le volume du trafic de fret ferroviaire tchèque est déjà nettement supérieur à la moyenne européenne, ce qui laisse entrevoir d'énormes problèmes dans un avenir proche (engorgement routier) et des besoins financiers conséquents à la fois pour l'extension des réseaux routier et ferré, l'aménagement des interconnexions rail-route et la création de plateformes de logistique intermodales. Selon des calculs effectués par l'IKB-Industriebank allemande, les investissements nécessaires pour l'ensemble des infrastructures est-européennes (rail et route) devraient dépasser les 275 milliards $€$, ce qui correspond grosso modo aux estimations de la Commission européenne. Pour l'économie tchèque, un meilleur raccordement de ses réseaux de transport de marchandises (route, rail et navigation fluviale) à l'Ouest est d'autant plus vital qu'il lui permettrait aussi d'optimiser ses échanges internationaux le long de l'Elbe jusqu'à la plateforme de Hambourg - son traditionnel port maritime.

L'Autriche limitrophe est, quant à elle, une zone de transit caractérisée, à l'intersection des grands axes européens nord-sud et est-ouest, qu'il soient routiers, ferroviaires ou fluviaux; les deux tiers des tonnes $/ \mathrm{km}$ recensés dans ce pays sont imputables aux échanges. Pour ce pays qui, conscient de sa position désormais centrale en Europe, mène une politique économique visant à attirer les grands groupes mondiaux, des infrastructures performantes sont vitales. Sa politique des transports suit donc une approche de corridors et de points nodaux : prolongement des grands axes vers les nouveaux Etats membres et extension des capacités intérieures. Dans le cadre de son schéma d'aménagement des transports (Generalverkehrsplan Österreich, GVP-Ö 2002) défini conjointement avec les Länder autrichiens et les sociétés opératrices, le gouvernement a ainsi programmé des investissements de 45,1 milliards € d'ici 2020.

La priorité actuelle est le désengorgement des réseaux routier et ferroviaire autour de Vienne (point nodal et plateforme intermodale avec l'axe du Danube) et l'extension des capacités vers la Hongrie et la Slovaquie. En projet dans ce contexte : la création d'une twin city Vienne/Bratislava grâce à l'intégration des réseaux. Le point nodal de Linz sera étendu vers la Bavière (Nuremberg) et la République tchèque (Brno, Prague). Autre priorité : la sécurité dans le tunnel du Brenner, sur le grand axe nord-sud du trafic ferroviaire de marchandises trans- 
alpin. Ces projets sont tous liés à divers programmes infrastructurels dont ils constituent en partie les prolongements, ainsi les programmes TINA des pays est-européens (géré par TINA-Vienna, à Vienne) et Réseaux trans-européens de transports (TEN-T) de I'UE. Reste, dans ce contexte, la question de l'indispensable aménagement du Danube (corridor Rhin-Main-Danube assurant la liaison intérieure entre la Mer du Nord et la Mer Noire) dont la réalisation achoppe toujours sur la farouche opposition des écologistes bavarois.

La Bavière se trouve, elle, confrontée moins à une question de goulots d'étranglement qu'à celle du désenclavement de ses marges septentrionales et orientales $(750 \mathrm{~km})$, trop longtemps coupées de leurs zones d'échanges à la fois par le Rideau de Fer et le Mur. Au sortir de la guerre, la Bavière rurale avait construit patiemment son dynamisme économique sur son raccordement aux pôles d'activités occidentaux, menant de front deux approches de développement structurel régional: la localisation d'entreprises industrielles et le développement des infrastructures. C'est ainsi que l'axe Danube-Main-Rhin avait été aménagé vers l'Ouest (port de Rotterdam). De l'autre côté du Rideau de Fer, le Danube devenait l'axe fluvial pour les industries est-européennes. Mais dans la région de Passau, contre le Rideau de Fer, l'interconnexion ne s'est jamais faite. Le fleuve, que les écologistes veulent préserver comme l'un des derniers biotopes européens avec la Loire, n'est toujours pas entièrement navigable. Le raccordement, pourtant programmé par les gouvernements bavarois, fédéral et ceux des Etats riverains (programme TEN-T) reste ainsi à l'état de projet.

Malgré sa politique de cohésion territoriale menée entre autres avec le soutien du Fonds structurel européen, la Bavière n'a pu ni dynamiser ni désenclaver ses régions orientales. Depuis la réunification, sa politique s'est en outre concentrée prioritairement, dans le cadre de la politique de reconstruction de l'économie et des infrastructures des nouveaux Länder, à construire ou moderniser, conjointement avec le Bund, les grands axes d'échanges avec la Saxe limitrophe au nord. Aujourd'hui, l'intégration des Etats est-européens peut constituer une nouvelle chance de développement pour cette Bavière orientale qui passe maintenant de la périphérie au centre. Le gouvernement du Land a donc lancé en novembre 2001 un «Programme de mise à niveau pour la Bavière orientale » (Ertüchtigungsprogramm Ostbayern) doté de 100 millions $€$. Destiné à dynamiser l'investissement ou la création d'entreprises, il comprend également un volet infrastructures dont, par exemple, l'extension de l'aéroport régional de Hof-Plauen qui desservira Bayreuth et Karlovy Vary.

Comme pour l'Autriche, l'accroissement attendu du trafic routier de marchandises (il pourrait quadrupler d'ici 2015) implique l'extension des infrastructures afin de réduire les goulots d'étranglement dans le réseau régional (compétence des Länder et communes) et de créer les grands axes pan-européens en coordination avec les programmes TEN-T et TINA (gérés par le Bund). Diverses autoroutes seront donc prolongées : Francfort-Vienne et Stuttgart-Munich-Vienne jusqu'à Budapest ou Paris-Nuremberg jusqu'à Prague. Des interconnexions sont également prévues. Pour le rail, sont programmées la modernisation de la liaison Nuremberg-Prague ou la création de liaisons interrégionales (Schwandorf-Domazlice, par exemple), sans oublier (sur le long terme) la liaison à haute vitesse reliant Paris à Budapest via Stuttgart, Munich et Vienne. Mais ces projets semblent aujourd'hui compromis. Le gouvernement fédéral, qui a vu fondre ses recettes prévisionnelles 2004 après l'échec du programme de péage routier Toll Collect, vient de réduire le montant de ses investissements dans les infrastructures de transport. Les sommes programmées seront ramenées de 9,5 milliards € par an à 7,7 milliards $€$ d'ici 2008. Ces restrictions risquent de retarder le désenclavement des régions orientales de Bavière, mais aussi l'intégration des réseaux bavarois et saxons (ICE Nuremberg-Erfurt-Berlin).

Elles auront également des répercussions sur l'aménagement des infrastructures saxonnes. Certes, avec $2700 \mathrm{~km}$ de voies ferrées, la Saxe dispose du
Bavière : désenclaver ses marges orientales...

... après le raccordement à la Saxe

Des liaisons transrégionales compromises?

Saxe : interconnexion ferroviaire avec le sud et l'est... 
... et des plateformes trimodales sur l'Elbe réseau ferroviaire le plus dense des Länder, mais il est encore insuffisamment relié aux grands axes vers le sud (Leipzig-Hof) ou le sud-ouest (Dresde-Nuremberg-Stuttgart-Karlsruhe). De même, la grande transversale est-ouest, le long de la «vallée de l'automobile » entre Dresde et Zwickau, a besoin de voir accroître ses capacités. Outre l'aménagement de ces infrastructures, l'extension de plusieurs plateformes intermodales est programmée : à Dresde, Zwikkau et Leipzig. Ces travaux font partie du programme lié à l'unité allemande (Verkehrsprojekte deutsche Einheit) et qui n'est pas encore achevé, alors que l'arrivée des nouveaux membres appelle, comme pour l'Autriche, à la fois l'aménagement des liaisons intérieures (le trafic de marchandises avec la République tchèque devrait plus que doubler) et leur prolongement vers l'Est. Deux chantiers sont néanmoins prévus : l'aménagement du tronçon Dresde-Pirna-frontière tchèque au sud-est (vers Prague) et l'extension du réseau entre Dresde et Görlitz à la frontière polonaise (et près de la frontière tchèque). Cette ville située sur la Neisse, coupée en deux par le Rideau de Fer, et dont la partie polonaise s'appelle Zgorzelec, était auparavant l'une des portes historiques vers l'Europe orientale. Elle devrait le redevenir.

Au plan des infrastructures routières, la Saxe mise sur la fluidité du trafic : desserte de l'aéroport de Leipzig, extension de l'autoroute de Dresde vers la Thuringe à l'Ouest et de celle de Chemnitz vers la Bavière. Mais le chantier prioritaire, pour ce Land comme pour la République tchèque, est la construction de l'autoroute Dresde-Prague. Pourtant, sa réalisation est ralentie du fait de l'opposition des écologistes tchèques: le tronçon reliant Breitenau (côté saxon) à Krásný Les (côté tchèque) traverse la forêt du nord de la Bohême. Elle pourrait se heurter également désormais à un problème de financement, les $50 \mathrm{~km}$ sur le sol allemand coûtant 562 millions $€$ à eux seuls. Indépendamment de ce dossier, la Saxe et la République tchèque ont décidé conjointement de développer des infrastructures trimodales, c'est-à-dire intégrant le rail, la route et la navigation fluviale. Car l'Elbe, qui prend sa source en République tchèque et traverse la Saxe sur 180 km, reliant Prague à Dresde, et qui débouche à Hambourg, est l'axe fluvial allemand le plus emprunté après le Rhin. Dès lors, du côté saxon, trois plateformes sont en cours d'aménagement, dont le port de Riesa au nordest de Dresde, de loin le plus important site intermodal en construction.

Peu À PEU, Les RÉSeaux S'InTERPÉnĖtrent. Mais, si on excepte l'Autriche, les politiques menées relèvent d'initiatives (inter-)régionales. Car à considérer le schéma national allemand d'aménagement (Bundesverkehrswegeplan) ou les programmes prioritaires adoptés par les Etats membres de l'UE dans le cadre du Réseau trans-européen de transport, l'absence de tracés visant à intégrer les dix nouveaux Etats membres est flagrante. Les schémas d'aménagement TINA et TEN-T coexistent parallèlement l'un à l'autre. II est vrai que l'UE 15 a fort à faire avec l'adaptation de son réseau 'intérieur' à l'état d'intégration du marché communautaire et que, de l'aveu même de la Commission, nombre de projets ont été retardés, faute de financement ou d'engagement nationaux suffisants (SPEECH/04/13). C'est aujourd'hui seulement qu'on semble comprendre l'enjeu des infrastructures de transport pour une politique de cohésion dans I'UE 25. Le gouvernement de Saxe - un Etat qui a intégré la RFA et l'UE 15 voici 14 ans seulement - parle en connaissance de cause lorsqu'il plaide pour l'adoption d'un «programme d'infrastructures lié à l'élargissement ». Sans politique de désenclavement et d'intégration à l'ouest, l'économie de Saxe n'aurait pas pu aussi vite dégager une dynamique de croissance qui, bien qu'imparfaite, en fait un challenger potentiel pour la Bavière, cet autre Etat qui, sans une vision globale d'aménagement du territoire, ne serait jamais devenu l'une des principales économies de l'UE - avec un PIB proche de celui des Pays-Bas.

Sites Internet : $\quad$ www.austria.gv.at ; www.bayern.de ; www.bmvbw.bund.de ; www.ikb.de ; www.sachsen.de ; www.tschechien.de ; www.europa.eu.int. 